\title{
Antibiotic Resistance Profile of Milk Samples from Cattle Mastitis
}

\author{
Shaista Shaheen ${ }^{1 *}$, Bilal Ahmad Bhat ${ }^{2}$, Aftab Ahmed Allaie ${ }^{2}$ and Gowhar Nabi Gora ${ }^{2}$ \\ ${ }^{1}$ Institute of Animal Health and Biological Products, Zakura, Srinagar, Animal Husbandry \\ Department, Kashmir, India \\ ${ }^{2}$ Disease Diagnostic Section, Institute of Animal Health and Biological Products, Zakura, \\ Srinagar, Department of Animal Husbandry, Kashmir, J \& K, India \\ *Corresponding author
}

\section{A B S T R A C T}

\begin{tabular}{|l|}
\hline Ke y w o r d s \\
Antibiotic \\
sensitivity test, \\
Mastitis, \\
Antibiogram, Milk \\
samples, Resistant \\
Antimicrobials \\
\hline Article Info \\
\hline $\begin{array}{l}\text { Accepted: } \\
\text { 12 August } 2020 \\
\text { Available Online: } \\
\text { 10 September } 2020\end{array}$ \\
\hline
\end{tabular}

The present study was undertaken to assess the antibiotic sensitivity pattern of the organisms from mastitic milk submitted from different districts viz., Srinagar, Ganderbal, Budgam, and Pulwama of union territory of Jammu and Kashmir, India. A total of 106 milk samples received by Institute of Animal Health and Biological Products, Zakura, Srinagar were subjected to in-vitro antibiotic sensitivity tests against the ten commonly used antimicrobials. Antibiogram studies showed highest resistance against Amoxycillin, Ampicillin, Cefipime, Doxycycline and Ceftriaxone while majority of the cultures showed susceptibility to Gentamicin, Amikacin and Ciprofloxacin. The present study reports a high resistance among Beta-Lactam group of antibiotics suggesting the non-judicious use of these drugs because of their wide spectrum of action. It is concluded that antibiotic sensitivity testing should be performed whenever possible before using antimicrobials so as to select the proper and sensitive antimicrobial drugs and avoid the indiscriminate use of antimicrobial drugs and prevent development of resistance among the micro-organisms.

\section{Introduction}

Mastitis is an inflammatory condition of udder of animals, which is characterized by the chemical and physical alterations in the milk (Blood and Radostits, 1989). Due to mastitis volume of milk produced decreases enormously thus causing great economic loss to the dairy industry (Sasidhar et al., 2002). There are two forms of mastitis viz. Clinical and sub-clinical, but sub-clinical form of mastitis is more important because it is more prevalent than clinical form and adversely affects both quality and quantity of milk produced (Seegers et al., 2003). The most common aetiological agents causing mastitis include E. coli, Staphylococcus spp, Streptococcus spp, Pseudomonas spp and Mycoplasma spp. Various antimicrobials are being used in the field to treat cases of mastitis. However indiscriminate use of antimicrobials without prior testing for 
selecting the specific antibiotic for a specific aetiological has led to the ineffective treatments and also results in the development of antimicrobial resistance. Antimicrobial resistance is one of the main reasons for the low milk production rates in dairy industry and also responsible for treatment failures (Barkema et al., 2006). The phenomenon of antimicrobial resistance is a worldwide cause of concern and is considered a great threat to both human health and food supply chain (Cosgrove and Carmeli, 2014). The main aim of the present study is the antibiotic resistance testing of milk samples received from individual farmers and field functionaries. This study will help us in the generation of antibiogram patterns which will be useful for stopping the ruthless use of antibiotics and will promote use of specific antibiotic for a specific aetiological agent.

\section{Materials and Methods}

A total of 106 milk samples from the cases of mastitis in cattle from the various districts viz., Srinagar, Ganderbal, Budgam, and Pulwama were received at Institute of Animal Health and Biological Products, Zakura, Srinagar, during the period September 2019July 2020. A loop of milk from each sample received was inoculated into the nutrient broth and incubated at temperature $37^{\circ} \mathrm{C}$ for 24 hours.

\section{Antibiotic sensitivity testing}

The antibiotic sensitivity test was performed as per Kirby-Bauer antibiotic disc diffusion method. The bacterial growth in nutrient broth was directly inoculated on Mueller-Hinton agar plates by means of cotton swabs to ensure confluent growth of organisms as per guidelines of Clinical and Laboratory Standards Institute, CLSI. Incubation of petridish layered with agar containing antibiotic disc was done at $37^{\circ} \mathrm{C}$ for 24 hours. The antibiotic susceptibility of the organism to different antimicrobial drugs was observed by measuring the diameter of zone of inhibitions (in $\mathrm{mm}$ ) and was compared with the standard chart (Fig.1). Based on this comparison the test organisms were determined to be resistant or susceptible to the antibiotic.

The milk cultures were tested for their sensitivity against 10 different antimicrobials which are in common use under field conditions. The antibiotic discs provided by the supplier (Himedia, Mumbai) were used in the study and included gentamicin $(50 \mu \mathrm{g})$, ciprofloxacin $(10 \mu \mathrm{g})$, amikacin $(30 \mu \mathrm{g})$, levofloxacin $(5 \mu \mathrm{g})$, ceftriaxone $(30 \mu \mathrm{g})$, doxycycline hydrochloride $(10 \mu \mathrm{g})$, amoxicillin (30 $\mathrm{g})$, ampicillin $(10 \mu \mathrm{g})$, enrofloxacin $(10 \mu \mathrm{g})$ and Cefipime/ Tazobactum $(30 \mu \mathrm{g})$. The concentration of antibiotic in each filter paper disc was as per the CLSI standards.

\section{Results and Discussion}

During the study period, a total of 106 mastitis milk samples were received and tested for antibiotic sensitivity. Out of total number of samples tested, sensitivity was observed in the descending order as Gentamicin (96.23\%), Amikacin (88.68\%) and Ciprofloxacin $(81.13 \%)$ while the resistance in descending was observed as Amoxycillin (96.23\%), Ampicillin (90.57\%), and Cefipime/tazobactam (83.02\%) (Table 1). In this study, the overall antibiogram profile indicated that gentamicin, amikacin and ciprofloxacin are the most effective antibiotics against micro-organisms responsible for the cases of mastitis. 
Table.1 Antibiotic sensitivity pattern of mastitis milk samples ( $\mathrm{n}=106)$

\begin{tabular}{|c|c|c|c|}
\hline S.No. & Antimicrobial & $\begin{array}{c}\text { No. of sensitive } \\
\text { samples }\end{array}$ & $\begin{array}{c}\text { Percentage of sensitive } \\
\text { samples }\end{array}$ \\
\hline $\mathbf{1}$ & Gentamicin & 102 & 96.23 \\
\hline $\mathbf{2}$ & Amikacin & 94 & 88.68 \\
\hline $\mathbf{3}$ & Ciprofloxacin & 86 & 81.13 \\
\hline $\mathbf{4}$ & Levofloxacin & 80 & 75.47 \\
\hline $\mathbf{5}$ & Enrofloxacin & 77 & 72.64 \\
\hline $\mathbf{6}$ & Ceftriaxone & 71 & 66.98 \\
\hline $\mathbf{7}$ & Doxycycline & 51 & 48.11 \\
\hline $\mathbf{8}$ & Cefipime/Tazobactam & 18 & 16.98 \\
\hline $\mathbf{9}$ & Ampicillin & 10 & 09.43 \\
\hline $\mathbf{1 0}$ & Amoxycillin & 04 & 03.77 \\
\hline
\end{tabular}

Fig.1

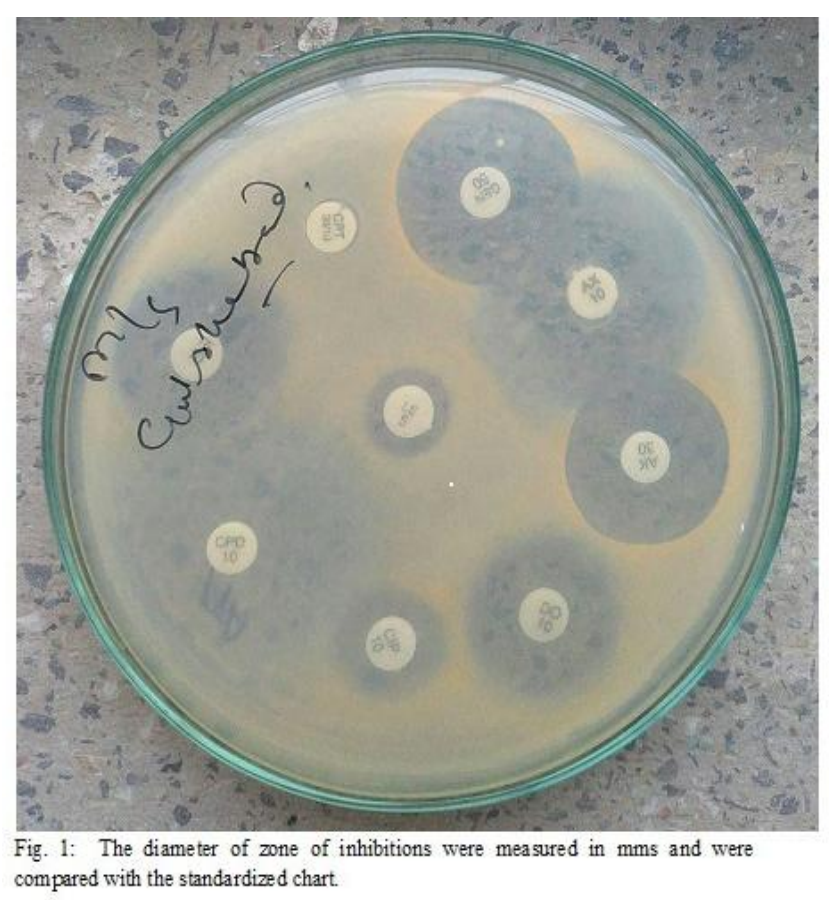

One of the leading causes for low milk production in India has been seen to be due to mastitis which is mostly of infectious nature (Deb et al., 2013). There are several reports of drug resistance in the common bacterial organisms responsible for mastitis because of indiscriminate use of antibiotics (Jeykumar $e t$ al., 2013). Many researchers have already reported multiple drug resistance in mastitis cases (Roychoudhury and Dutta, 2009; Chavan et al., 2007). The long term use of third generation cephalosporins has led to the occurrence of resistance against mastitis causing bacteria (Moon et al., 2007). Judicial use of antimicrobials can effectively lessen the problem of multiple drug resistance. This study was carried out to perform antimicrobial susceptibility tests to choose 
most appropriate antimicrobial agents for effective and rationale treatment of mastitis cases.

Our study showed highest susceptibility towards gentamicin and amikacin which is in close agreement with many workers (Ranjan et al., 2010; Sarangi et al., 2009; Moges et al., 2011). Gentamicin was reported to be the most effective drug among the antimicrobials used by many researchers (Sumathi et al., 2008). For selecting the most appropriate antibiotic and to have updated knowledge with respect to changing trends of antibiotic resistance, regular testing of milk cultures for determining the susceptibility patterns is highly useful and important. This practice will help us in developing antibiotic usage policy at the local level and will also contribute towards avoiding the indiscriminate use of antibiotics which are no longer effective in treating the cases of mastitis. The present study showed a high percentage of resistance against most commonly used antibiotics and gentamicin proved to be drug of choice in majority of antibiotic sensitivity tests conducted.

One of the important things to restrict the development of multidrug resistance will be generation of awareness among the animal owners and field functionaries for reducing the unnecessary use of antimicrobial agents. As mastitis is considered as a leading cause of antimicrobial usage in farm animals, especially in dairy sector, quick diagnosis and generation of correct antibiogram is very essential to prevent spread of resistant microorganisms to other susceptible animals.

In conclusion, this study showed the highest resistance against the Beta-Lactam group of antibiotics which are used irrationally because of their broad spectrum of action. It is therefore important that antibiotic sensitivity testing should be made a regular practice so that the indiscriminate use of antibiotics can be avoided and development of antibiotic resistance among micro-organisms can be minimized.

\section{Acknowledgement}

The authors are grateful to the Director, Animal Husbandry Department Kashmir, for support and encouragement and providing all the necessary facilities for this study.

\section{Conflict of Interest}

The authors declare that they have no competing interest.

\section{References}

Barkema HW, Schukken YH, Zadoks RN. 2006. Invited review: The role of cow, pathogen, and treatment regimen in the therapeutic success of bovine mastitis. J. Dairy Sci. 89: 1877-1895. doi:http://dx.doi.org/10.3168/jds.S002 2-0302(06)72256-1

Blood, D.C. and Radostits, O.M.1989. Veterinary Medicine: A textbook of the Diseases of Cattle, Sheep, Pigs, Goats and Horses, $7^{\text {th }}$ ed., 501.

Chavan VV, Digraskar SU, Dhonde SN, et al., 2007. Observation on bubaline subclinical mastitis in and around Parbhani. Indian J. Field Vet. 3:50.

Clinical and laboratory Standards Institute Document M100-S23. Vol 33, No.1. USA. Wayn PA. 2013. Performance standards for antimicrobial susceptibility testing: twenty first information supplement.

Cosgrove, S.E and Carmeli, Y. The impact of antimicrobial resistance on health and economic outcomes. Clin. Infect. Dis. 1433(36), 1433-7 (2014)

Deb R, Kumar A, Chakraborty S, et al., 2013. Trends in diagnosis and control of 
bovine mastitis: A review. Pak. J. Biol. Sci. 16(23): 1653-1661. doi:10.3923/pjbs.2013.1653.1661.

Jeykumar M, Vinodkumar G, Bashir BP, et al 2013. Antibiogram of mastitis pathogens in the milk of crossbred cows in Namakkal district, Tamil Nadu. Vet. World 6(6): 354-356. doi: 10.5455/vetworld.2013.354-356.

Moges N, Asfaw Y, Belihu K, et al., 2011. Antimicrobial susceptibility of mastitis pathogen from small holder dairy herd in and around Gonder, Ethopia. J. Anim. Vet. Adv. 10(12):1616-1622.

Moon JS, Lee AR, Kang HM, et al. 2007. Phenotypic and genetic antibiogram of methicillin resistant Staphylococci isolated from bovine mastitis in Korea. J. Dairy Sci. 90: 1176-1185. doi:10.3168/jds.S00220302(07)71604-1.

Ranjan R, Gupta MK, Singh S, et al., 2010. Current trend of drug sensitivity in bovine mastitis. Vet. World 3(1):1720.
Roychoudhury P, Dutta, TK. 2009. Prevalence and antibiotic sensitivity pattern of bacteria from bovine mastitis in Mizoram. Indian J. Anim. Sci.79: 483-484.

Sarangi LN, Panda HK, Priyadarshini A, et al.2009. Antibiogram and drug resistance of Staphylococcus aureus isolated from bovine clinical and subclinical mastitis. Journal of Research, 27(1-2): 136-138.

Sasidhar P V K, Ramana Reddy Y and Sudhakar Rao G V 2002 Economics of mastitis, Indian Journal of Veterinary Science 72 (6): 439-440.

Seegers, H., Fourichon, $\mathrm{C}$ and Beaudeau, F. 2003. Production effects related to mastitis and mastitis economics in dairy cattle herds. Veterinary Research. 34: 475-491.

Sumathi BR, Veeregpwda BM, Amitha RG. 2008. Prevalence and antimicrobial profile of bacterial isolates from clinical mastitis. Veterinary World1: 237-238.

\section{How to cite this article:}

Shaista Shaheen, Bilal Ahmad Bhat, Aftab Ahmed Allaie and Gowhar Nabi Gora. 2020. Antibiotic Resistance Profile of Milk Samples from Cattle Mastitis. Int.J.Curr.Microbiol.App.Sci. 9(09): 1299-1303. doi: https://doi.org/10.20546/ijcmas.2020.909.159 